\title{
DEGUM-Mitteilungen
}

Deutsche Gesellschaft für Ultraschall in der Medizin



Bauchaortenaneurysmen

\section{Das Ultraschall-Screening für Männer ab 65 kommt - DEGUM begrüßt neue Richtlinie}

Berlin, Juni 2017 - Gute Neuigkeiten aus der Gesundheitspolitik: Gesetzlich krankenversicherte Männer ab 65 Jahren haben künftig Anspruch auf ein einmal durchgeführtes Ultraschall-screening zur Früherkennung von Bauchaortenaneurysmen. Die entsprechende Richtlinie des Gemeinsamen Bundesausschusses (G-BA) ist nun zusammen mit einer Versicherteninformation in Kraft getreten. Die Deutsche Gesellschaft für Ultraschall in der Medizin e. V. (DEGUM) sieht das als wichtigen Schritt an - empfiehlt jedoch auch Patienten mit einem besonderen Risikoprofil wie etwa Diabetes oder Rauchen sowie Frauen einzubeziehen.
Die Ruptur eines Bauchaortenaneurysmas zählt zu den zehn häufigsten Todesursachen. „Das Tückische an der Erkrankung liegt darin, dass die Aussackung des größten Gefäßes im Bauchraum - dem sogenannten Aneurysma -, meist keine Beschwerden verursacht", sagt Professor Dr. med. Thomas Fischer, stellvertretender Leiter der Sektion Radiologie bei der DEGUM und Leiter des Interdisziplinären Ultraschall-Zentrums am Institut für Radiologie am Campus Charité Berlin-Mitte. „Die Betroffenen ahnen nichts von der Gefahr, in der sie sich befinden." Reiße die Bauchaorta jedoch, könne der Patient innerhalb kurzer Zeit innerlich verbluten.
„Mittels Ultraschall ist ein Bauchaortenaneurysma von einem erfahrenen Untersucher jedoch leicht bei einer Früherkennungsuntersuchung zu diagnostizieren“, sagt Professor Fischer. Dabei misst der Arzt mit einem Ultraschallgerät den Durchmesser des Blutgefäßes: „Bei einem Durchmesser ab 5,5 Zentimetern ist das Risiko für ein Reißen des Gefäßes recht hoch, sodass wir den Patienten dann zu einem operativen Eingriff raten“, berichtet der DEGUM-Experte. Bei kleineren Aneurysmen sei es empfehlenswert, regelmäßige Kontrolluntersuchungen durchzuführen. So könne überprüft werden, ob sich diese weiter ausdehnen. Wenn das der Fall sei, würde gegebenenfalls ein minimalinvasiver oder offe- 
ner operativer Eingriff durchgeführt - und so möglicherweise das Leben eines Betroffenen gerettet. Für das Screening sollten Untersucher eine nachweisbare Qualifikation haben, beispiels-weise ein DEGUM-Zertifikat der Stufe 1, empfiehlt der Experte. Denn es gehe nicht nur darum, den Durchmesser der Bauchorta zu bestimmen, sondern auch einen Einriss oder ein Aneurysma des Gefäßes frühzeitig zu erkennen.

Auch wenn Männer häufiger von einem Bauchaortenaneurysma betroffen seien als Frauen, sollten nach Ansicht der Ultraschall-Experten auch sie von der Vorsorgeuntersuchung profitieren können. „Darüber hinaus wäre es ratsam, auch Patienten mit einem besonderen Risikoprofil - beispielsweise Personen mit einer Fettstoffwechselerkrankung, Diabetes, Bluthochdruck und starke Raucher ab dem 55. Lebensjahr - in das Vorsorgescreening einzuschließen“, sagt Professor Fischer.

Bis das Screeningangebot zur Früherkennung eines Bauch-aortenaneurysmas auf Krankenschein von Männern ab 65 Jahren wahrgenommen werden kann, dauert es jetzt noch bis zu sechs Monaten: Der zuständige Bewertungsausschuss muss zunächst noch die Frage der ärztlichen Vergütung regeln und hat dafür bis zu einem halben Jahr Zeit.

\section{Weiterführende Information}

Richtlinie „Ultraschallscreening auf Bauchaortenaneurysmen“ https://www.g-ba.de/downloads/62-4921411/US-BAA-RL_2016-10-20_2017-0316_iK-2017-06-10.pdf

\section{Versicherteninformation} https://www.g-ba.de/downloads/17-984330/2017_03_16_G-BA_Merkblatt_Versicherteninformation-Bauchaortenaneurysmen_bf.pdf

\section{ÜBER DIE DEGUM}

Die Deutsche Gesellschaft für Ultraschall in der Medizin (DEGUM) bietet ein Forum für den wissenschaftlichen und praktischen Erfahrungsaustausch auf dem Gebiet des medizinischen
Ultraschalls. Sie vereint rund 10000 Ärzte verschiedener Fachgebiete, medizinische Assistenten, Naturwissenschaftler und Techniker. Ultraschalldiagnostik ist heute das am häufigsten eingesetzte bildgebende Verfahren in der Medizin. Ultraschallanwendern bescheinigt die DEGUM eine entsprechende Qualifikation mit einem Zertifikat der Stufen I bis III. DEGUM zertifizierte Ärzte finden Patienten im Internet unter: www.degum.de

\section{Kontakt für Rückfragen:}

Friederike Gehlenborg

Deutsche Gesellschaft für Ultraschall in der Medizin (DEGUM)

Pressestelle

Postfach 301120

70451 Stuttgart

Tel.: $07118931-295$

Fax: $07118931-167$

E-mail: gehlenborg@medizinkommunikation.org 